\title{
On algebraic properties of general proper decentralized systems
}

\author{
Runyi $\mathrm{Yu}$
}

Letter Box 29, Department of Automation, Beijing Institute of Chemical Technology, Beijing 100029, China

\author{
M. Erol Sezer
}

Department of Electrical and Electronics Engineering, Bilkent University, 06533 Ankara, Turkey

\section{Weibing Gao}

The Seventh Research Division, Beijing University of Aeronautics and Astronautics, Beijing 100083, China

Received 2 November 1992

Revised 16 February 1993

\begin{abstract}
The new concepts of the decentralized output feedback variable polynomial, the decentralized output feedback cycle index of general proper systems, and the geometric multiplicities of decentralized fixed modes are introduced. Their computational methods and some algebraic properties are presented. It is shown that the decentralized output feedback cycle index of a general proper system is equal to one when the system has no fixed modes or equal to the maximum of the geometric multiplicities of its decentralized fixed modes. It is also shown that almost all decentralized output feedback can be used to make the zeros of the decentralized variable polynomial distinct, and disjoint from any given finite set of points on the complex plane.
\end{abstract}

Keywords: Decentralized systems; control system analysis; output feedback; fixed modes; algebraic properties.

\section{Introduction}

There is continuing interest in the study of decentralized control systems. The reason for this interest follows since many control problems of modern industrial society are subject to information flow constraint. Recently, Davison and Chang [3] have studied the decentralized stabilization and pole assignment problem for a general proper system, which can be described by

$$
\begin{aligned}
& \dot{x}=A x+\sum_{i=1}^{N} B_{i} u_{i}, \\
& y_{i}=C_{i} x+\sum_{j=1}^{N} D_{i j} u_{j}, \quad i \in \mathscr{N},
\end{aligned}
$$

where $x \in \mathscr{R}^{n}$ is the state of the system, $u_{i} \in \mathscr{R}^{r_{i}}$ and $y_{i} \in \mathscr{R}^{m_{i}}$ are the input and output vectors of the $i$ th control channel, respectively, and $A, B_{i}, C_{i}, D_{i j}, i, j \in \mathscr{N}=\{1,2, \ldots, \mathrm{N}\}$, are real constant matrices of appropriate sizes. Moreover, it is assumed that $B_{i}, C_{i}$ are of full rank and system (1) is wholly controllable and observable.

According to [3], there are three main motivations to study general proper decentralized control systems. The first one arises from a study of large-scale systems which are modelled by descriptor state-space models. The second one is that any periodic linear discrete-time system can always be represented as a discrete 
time-invariant system with direct feedthrough. The third motivation is that many large-scale systems often have a simplified model with a representation given by (1). For this class of systems, Davison and Chang [3] and Vaz and Davison [7] obtained some characterization results and invariant properties of decentralized fixed modes, which play a key role in analysis and design of decentralized control problems. Davison and Chang [3] further showed that system (1) can be assigned a closed-loop spectrum using linear time-invariant decentralized controllers only if the spectrum includes the set of decentralized fixed modes.

The aim of this paper is to answer the question of what can be said about the resultant closed-loop system

$$
\dot{x}=\left(A+B(I-K D)^{-1} K C\right) x
$$

after decentralized static output feedbacks

$$
u_{i}=K_{i} y_{i}, \quad i \in \mathcal{A},
$$

are applied to (1), where $B=\left(B_{1} \cdots B_{N}\right), C=\left(C_{1}^{\mathrm{T}} \cdots C_{N}^{\mathrm{T}}\right)^{\mathrm{T}}, D=\left(D_{i j}\right), i, j \in \mathscr{N}$, and $K \in \mathscr{K} \subset \mathscr{R}^{r \times m}$, with $r=\sum_{i=1}^{N} r_{i}, m=\sum_{i=1}^{N} m_{i}$, and $\mathscr{K}=\left\{K \mid K=\operatorname{diag}\left(K_{1} \cdots K_{N}\right), K_{i} \in \mathscr{R}^{r_{i} \times m_{i}}, i \in \mathscr{N}, \operatorname{det}(I-K D) \neq 0\right\}$.

This work is an extension of [12], which considers the same problem for system (1) with $D=0$. It should be noted that this extension is not just trivial. The results here depend critically on the values of the $D$ parameters.

\section{Preliminary knowledge}

Let $x \in \mathscr{R}^{n}$ and let $f(x)$ denote a polynomial in the ring of polynomials $\mathscr{R}[x]$. A set $\mathscr{V}$ in $\mathscr{R}^{n}$ is called a robust set (or robust) if there is a polynomial $f(x) \in \mathscr{R}[x]$, such that

$$
\mathscr{N}=\mathscr{R}^{n}-\mathscr{N}(f), \quad \mathscr{N}(f)=\left\{x \mid f(x)=0, x \in \mathscr{R}^{n}\right\},
$$

where $f(x)$ is not identically equal to zero and $\mathscr{N}(\cdot)$ denotes the set of zeros of polynomial ". ". Obviously, if $f(x)$ is a nonzero constant number, then $\mathscr{V}=\mathscr{R}^{n}$. Stating that $V$ is a robust set is equivalent to stating that almost all points in $\mathscr{R}^{n}$ belong to $\mathscr{r}$. Also the union and intersection of any finite robust sets are still robust.

Lemma 2.1 (Bocher [1]). Let

$$
\begin{aligned}
& f(x)=a_{n} x^{n}+a_{n-1} x^{n-1}+\cdots+a_{1} x+a_{0}, \quad n>0, \\
& g(x)=b_{m} x^{m}+b_{m-1} x^{m-1}+\cdots+b_{1} x+b_{0}, \quad m \geq 0,
\end{aligned}
$$

be polynomials in $\mathscr{R}[x]$. Let $r\left(a_{n}, \ldots, a_{0} ; b_{m}, \ldots, b_{0}\right)$, a polynomial in $\mathscr{R}\left[a_{n}, \ldots, a_{0} ; b_{m}, \ldots, b_{0}\right]$, denote the resultant of $f(x)$ and $g(x)$. Then a necessary and sufficient condition under which the two polynomials $f(x)$ and $g(x)$ have no common zeros is that $r\left(a_{n}, \ldots, a_{0} ; b_{m}, \ldots, b_{0}\right)$ is nonzero.

Lemma 2.2. If $(A, B)$ is controllable and $(A, C)$ observable, then the set

$$
\mathscr{K}^{\prime}=\left\{\mathscr{K} \mid \text { the eigenvalues of } A+B(I-K D)^{-1} K C \text { are distinct, } \operatorname{det}(I-K D) \neq 0, K \in \mathscr{R}^{r \times m}\right\}
$$

is robust.

Proof. From [5], the controllability of $(A, B)$ and the observability of $(A, C)$ imply that $A+B \tilde{K C}$ has $n$ distinct eigenvalues for almost all $\tilde{K} \in \mathscr{R}^{r \times m}$. Choose $\tilde{K}$ so that $I-D \tilde{K}$ and $I+D \tilde{K}$ are nonsingular and $A+B \tilde{K} C$ has $n$ distinct eigenvalues, and let $K=\tilde{K}(I+D \tilde{K})^{-1}$. We get $A+B(I-K D)^{-1} K C=A+B \tilde{K} C$. Thus, $\mathscr{K}^{\prime}$ is not empty.

Now, let

$$
\operatorname{det}\left(s I-A-B(I-K D)^{-1} K C\right)=\frac{f(s, K)}{d(K)},
$$


where $d(K)=\operatorname{det}(I-K D)$ and $f(s, K)=s^{n}+a_{n-1}(K) s^{n-1}+\cdots+a_{0}(K)$, with $a_{i}(K), i=0,1, \ldots, n-1$, being polynomials of $\{k(i, j)\}$ - the $(i, j)$ th element of $K$;

$$
g(s)=\frac{\mathrm{d}}{\mathrm{d} s} f(s)=n s^{n-1}+(n-1) a_{n-1}(K) s^{n-2}+\cdots+a_{1}(K),
$$

then the resultant of $f(s)$ and $g(s)$ is a polynomial of $\{k(i, j)\}$, which is denoted by $r(K)$. It is clear from Lemma 2.1 that

$$
\mathscr{K}^{\prime}=\mathscr{R}^{q}-\mathscr{N}(d(K) r(K)),
$$

where $q=r \times m$. Since $\mathscr{K}^{\prime}$ is not empty, the polynomial $d(K) r(K)$ is not identically equal to zero. Therefore, the set $\mathscr{K}^{\prime}$ is robust.

Lemma 2.3. Let $A \in \mathscr{R}^{n \times l}, B \in \mathscr{R}^{n \times r}$, and $C \in \mathscr{R}^{m \times l}$ be constant matrices, $B, C$ be of full rank, 2 be any given set containing a robust set in $\mathscr{R}^{r \times m}$; then

$$
\max \{\operatorname{rank}(A+B K C), K \in \mathscr{Q}\}=\min \left\{\operatorname{rank}[A, B], \operatorname{rank}\left[\begin{array}{l}
A \\
C
\end{array}\right]\right\} .
$$

Proof. Xie and Jing [10] have shown that the above equality is true if $\mathscr{Q}=\mathscr{R}^{r \times m}$. We can further prove that

$$
\mathscr{Q}^{\prime}=\left\{K \mid \operatorname{rank}(A+B K C)=\gamma, K \in \mathscr{R}^{r \times m}\right\}
$$

is a robust set, where

$$
\gamma=\min \left\{\operatorname{rank}[A, B], \operatorname{rank}\left[\begin{array}{l}
A \\
C
\end{array}\right]\right\} .
$$

By noting that $\operatorname{rank}(A+B K C) \leq \gamma$ for any $K \in \mathscr{Q}$, the result then follows from the robustness of the intersection of $\mathscr{Q}^{\prime}$ and the robust set contained in $\mathscr{Q}$.

\section{Main results}

\subsection{Definitions}

To discuss algebraic properties of general proper decentralized control systems, we first introduce the following definitions, which, unlike the decentralized fixed polynomial and fixed modes, characterize the effectiveness of decentralized output feedbacks.

Definition 3.1. The decentralized output feedback variable polynomial (DVP) of the general proper decentralized control system (GDS) (1) with respect to (w.r.t.) $\mathscr{K}$ is defined as

$$
p_{\mathrm{v}}(s, A, B, C, D, K)=\frac{\operatorname{det}\left(s I-A-B(I-K D)^{-1} K C\right)}{p_{\mathrm{f}}(s, A, B, C, D, \mathscr{K})},
$$

where $p_{\mathrm{f}}(s, A, B, C, D, \mathscr{K})$ is the decentralized fixed polynomial of GDS (1) w.r.t. $\mathscr{K}$. For brevity, we will use the notation $p_{\mathrm{v}}(s, K), p_{\mathrm{f}}(s, \mathscr{K})$ to denote $p_{\mathrm{v}}(s, A, B, C, D, K)$ and $p_{\mathrm{f}}(s, A, B, C, D, \mathscr{K})$, respectively, in the following derivations.

The above definition can also be used for determination of DVP, since algorithms for determination of decentralized fixed modes are available. 
Definition 3.2. Consider GDS (1); the number

$$
k=\min \left\{\operatorname{cyc}\left(A+B(I-K D)^{-1} K C\right), K \in \mathscr{K}\right\}
$$

is called the decentralized output feedback cycle index (DCI) of the GDS w.r.t. $\mathscr{K}$, and is denoted by $\operatorname{cyc}(A, B, C, D, \mathscr{K})$, where $\operatorname{cyc}(\cdot)$ is the cycle index of matrix ".".

It is obvious that $\operatorname{cyc}(A, B, C, D, \mathscr{K}) \leq \operatorname{cyc}(A)$.

Definition 3.3. Let $\operatorname{DFM}(A, B, C, D, \mathscr{K})$ be the set of decentralized fixed modes (DFM) of GDS (1), $\lambda \in \operatorname{DFM}(A, B, C, D, \mathscr{K})$; then the number

$$
m_{\lambda}=\min \left\{\operatorname{dim} \mathscr{X}_{K \lambda}, K \in \mathscr{K}\right\}
$$

is called the geometric multiplicity $(\mathrm{GM})$ of the fixed mode $\lambda$, where $\mathscr{X}_{K \lambda}=\left\{x \mid\left(A+B(I-K D)^{-1}\right.\right.$ $\left.K C-\lambda I) x=0, x \in \mathscr{R}^{n}\right\}$ and $\operatorname{dim} \mathscr{X}_{K \lambda}$ is the dimension of the subspace $\mathscr{X}_{K \lambda}$.

\subsection{Separation of zeros of DVP}

Theorem 3.4. The set

$$
\mathscr{K}_{1}=\left\{K \mid \text { the zeros of } p_{v}(s, K) \text { are distinct, } K \in \mathscr{K}\right\}
$$

is robust.

Proof. First, we prove that the set $\mathscr{K}_{1}$ is not empty. If this is not true, i.e., for every $K \in \mathscr{K}$, there is always a multiple zero $s_{0}(K)$ of $p_{v}(s, K)$, then there exists a polynomial $p_{3}(s, K)$ such that

$$
p_{v}(s, K)=\left(s-s_{0}(K)\right)^{2} p_{3}(s, K),
$$

where $s_{0}(K)$ is a function of $K$ and is not a constant. Without loss of generality, suppose $s_{0}(K)$ is dependent on $K_{N}$; there must exist parameters $K_{1}^{0}, \ldots, K_{N-1}^{0}$ such that $s_{0}\left(K_{1}^{0}, \ldots, K_{N-1}^{0}, K_{N}\right)$ is dependent on $K_{N}$ and is not a constant.

Now

$$
\operatorname{det}\left(s I-\bar{A}_{N}\right)=p_{\mathrm{f}}(s)\left(s-s_{0}\left(K_{1}^{0}, \ldots, K_{N-1}^{0}, K_{N}\right)\right)^{2} p_{3}\left(s, K_{1}^{0}, \ldots, K_{N-1}^{0}, K_{N}\right),
$$

where $\bar{A}_{N}=\left.\left(A+B(I-K D)^{-1} K C\right)\right|_{K_{l}=K_{l}^{0}, l=1, \ldots, N-1}$.

On the other hand, it can be shown that

$$
\begin{aligned}
\operatorname{det}\left(s I-\bar{A}_{N}\right) & =\operatorname{det}\left(s I-\bar{A}_{N-1}-\bar{B}_{N-1}\left(I-K_{N} \bar{D}_{N-1}\right)^{-1} K_{N} \bar{C}_{N-1}\right) \\
& =p_{\mathrm{f}}\left(s, \bar{A}_{N-1}, \bar{B}_{N-1}, \bar{C}_{N-1}, \bar{D}_{N-1}, \mathscr{K}_{N}\right) p_{v}\left(s, \bar{A}_{N-1}, \bar{B}_{N-1}, \bar{C}_{N-1}, \bar{D}_{N-1}, K_{N}\right),
\end{aligned}
$$

where

$$
\begin{aligned}
& \bar{A}_{N-1}=A+B^{N-1}\left(I-K_{0}^{N-1} D^{N-1}\right)^{-1} K_{0}^{N-1} C^{N-1}, \\
& \bar{B}_{N-1}=B_{N}+B^{N-1}\left(I-K_{0}^{N-1} D^{N-1}\right)^{-1} K_{0}^{N-1}\left[\begin{array}{c}
D_{1 N} \\
\vdots \\
D_{N-1 N}
\end{array}\right], \\
& \bar{C}_{N-1}=C_{N}+\left(D_{N 1} \cdots D_{N N-1}\right)\left(I-K_{0}^{N-1} D^{N-1}\right)^{-1} K_{0}^{N-1} C^{N-1}, \\
& \bar{D}_{N-1}=D_{N N}+\left(D_{N 1} \cdots D_{N N-1}\right)\left(I-K_{0}^{N-1} D^{N-1}\right)^{-1} K_{0}^{N-1}\left[\begin{array}{c}
D_{1 N} \\
\vdots \\
D_{N-1 N}
\end{array}\right], \\
& \mathscr{K}_{N}=\left\{K_{N} \mid \operatorname{det}(I-K D) \neq 0, K=\operatorname{diag}\left(K_{1}^{0} \cdots K_{N-1}^{0} K_{N}\right)\right\},
\end{aligned}
$$


with

$$
\begin{aligned}
& B^{N-1}=\left(B_{1} \cdots B_{N-1}\right), \quad C^{N-1}=\left(C_{1}^{\mathrm{T}} \cdots C_{N-1}^{\mathrm{T}}\right)^{\mathrm{T}}, \\
& D^{N-1}=\left(D_{i j}\right), \quad i, j=1, \ldots, N-1, \quad K_{0}^{N-1}=\operatorname{diag}\left(K_{1}^{0} \cdots K_{N-1}^{0}\right),
\end{aligned}
$$

and $p_{\mathrm{f}}\left(s, \bar{A}_{N-1}, \bar{B}_{N-1}, \bar{C}_{N-1}, \bar{D}_{N-1}, \mathscr{K}_{N}\right)$ is the fixed polynomial of $\left(\bar{A}_{N-1}, \bar{B}_{N-1}, \bar{C}_{N-1}, \bar{D}_{N-1}\right)$ w.r.t. $\mathscr{K}_{N}$, $p_{\mathrm{v}}\left(s, \bar{A}_{N-1}, \bar{B}_{N-1}, \bar{C}_{N-1}, \bar{D}_{N-1}, K_{N}\right)=\operatorname{det}\left(s I-\bar{A}_{N-1}^{\text {co }}-\bar{B}_{N-1}^{\text {co }}\left(I-K_{N} \bar{D}_{N-1}\right)^{-1} \bar{C}_{N-1}^{\text {co }}\right), \quad$ with $\quad\left(\bar{A}_{N-1}^{\text {co }}, \bar{B}_{N-1}^{\text {co }}\right.$, $\left.\bar{C}_{N-1}^{\text {co }}\right)$ being the controllable and observable part of the triple $\left(\bar{A}_{N-1}, \bar{B}_{N-1}, \bar{C}_{N-1}\right)$. From Lemma 2.2 , we know that the zeros of $p_{v}\left(s, K_{N}\right)$ can be assigned as distinct. This contradicts the assumption that $s_{0}(K)$ is a multiple zero.

The rest of the proof is exactly the same as the last part of the proof of Lemma 2.2 , except that $q=$ $\sum_{i=1}^{N} r_{i} \times m_{i}$ here.

\subsection{Shifting of zeros of DVP}

Theorem 3.5. For any given finite set $P=\left\{p_{1}, \ldots, p_{l}\right\} \subset \mathscr{C}$, the set

$$
\mathscr{K}_{2}=\left\{K \mid P \cap \mathscr{N}\left(p_{\mathrm{v}}(s, A, B, C, D, K)\right)=\emptyset, K \in \mathscr{K}\right\}
$$

is a robust set.

Proof. Define

$$
\mathscr{K}(i)=\left\{K \mid p_{i} \notin \mathscr{N}\left(p_{\mathrm{v}}(s, A, B, C, D, K)\right), K \in \mathscr{K}\right\} .
$$

By definition of $p_{\mathrm{v}}(A, B, C, D, K)$, the set $\mathscr{K}(i)$ is not empty, and it is easy to see that $\mathscr{K}(i)$ is also robust. Finally, noting that

$$
\mathscr{K}_{2}=\bigcap_{i=1}^{N} \mathscr{K}(i)
$$

we get that the set $\mathscr{K}_{2}$ is robust.

As an immediate corollary of Theorem 3.5, we have that in the case of $\operatorname{DFM}(A, B, C, D, \mathscr{K})=\emptyset$, almost all decentralized output feedback can make the spectrum of the resultant closed-loop system disjoint from any finite set of points on the complex plane.

\subsection{Determination of geometric multiplicities of DFM}

Theorem 3.6. Let $\lambda$ be a decentralized fixed mode of system (1); then its geometric multiplicity is given by

$$
m_{\lambda}=\max \left\{n-\operatorname{rank}\left[\begin{array}{cc}
\lambda I-A & B_{\mathscr{S}} \\
C_{\mathscr{S}^{1}} & D_{\mathscr{S}^{+} \mathscr{S}}
\end{array}\right], \mathscr{S} \subset \mathscr{N}\right\},
$$

where $\quad \mathscr{P}=\left\{i_{1}, \ldots, i_{s}\right\} \subset \mathcal{N}, \quad \mathscr{S}^{\perp}=\mathscr{N}-\mathscr{S}=\left\{j_{1}, \ldots, j_{N-s}\right\}, \quad$ and $\quad B_{\mathscr{S}}=\left(B_{i_{1}} \cdots B_{i_{s}}\right) ; \quad C_{\mathscr{P}^{\perp}}=$ $\left(C_{j_{1}}^{\mathrm{T}} \cdots C_{j_{N-s}}^{\mathrm{T}}\right)^{\mathrm{T}}, D_{\mathscr{S} \perp \mathscr{S}}=\left(D_{\alpha \beta}\right), \alpha \in \mathscr{S}^{\perp}, \beta \in \mathscr{S}$.

Proof. According to Definition 3.3,

$$
\begin{aligned}
m_{\lambda} & =\min \left\{\operatorname{dim} \mathscr{X}_{K \lambda}, K \in \mathscr{K}\right\} \\
& =\min \left\{\operatorname{dim} \operatorname{ker}\left(\lambda I-A-B(I-K D)^{-1} K C\right), K \in \mathscr{K}\right\} \\
& =\min \left\{n-\operatorname{rank}\left(\lambda I-A-B(I-K D)^{-1} K C\right), K \in \mathscr{K}\right\} \\
& =n-\max \left\{\operatorname{rank}\left(\lambda I-A-B(I-K D)^{-1} K C\right), K \in \mathscr{K}\right\} .
\end{aligned}
$$


Choose $\theta$ now so that $\theta \neq 0, \theta \notin \operatorname{sp}(A)$, and let $K=\bar{K} / \theta$; we have

$$
\begin{aligned}
\max & \left\{\operatorname{rank}\left(\lambda I-A-B(I-K D)^{-1} K C\right), K \in \mathscr{K}\right\} \\
& =\max \left\{\operatorname{rank}\left(\lambda I-A-B(\theta I-K D)^{-1} K C\right), K \in \overline{\mathscr{K}}\right\},
\end{aligned}
$$

where $\overline{\mathscr{K}}=\left\{K \mid K=\operatorname{diag}\left(K_{1}, \ldots, K_{N}\right), K_{i} \in \mathscr{R}^{r_{i} \times m_{i}}, i \in \mathcal{N}, \operatorname{det}(\theta I-K D) \neq 0\right\}$.

Now

$$
\begin{aligned}
& \operatorname{rank}\left(\lambda I-A-B(\theta I-K D)^{-1} K C\right) \\
& \quad=\operatorname{rank}\left[\begin{array}{cc}
\lambda I-A & B \\
K C & \theta I_{r}-K D
\end{array}\right]-r \\
& \quad=\operatorname{rank}\left(\left[\begin{array}{cc}
\lambda I-A & B \\
0 & \theta I_{r}
\end{array}\right]-\left[\begin{array}{c}
0 \\
I_{r}
\end{array}\right] K\left[\begin{array}{ll}
C & D
\end{array}\right]\right)-r \\
& \quad=\operatorname{rank}\left(\left(\left[\begin{array}{cc}
\lambda I-A & B \\
0 & \theta I_{r}
\end{array}\right]-\sum_{i=1}^{N-1} I^{i} K_{i}\left[\begin{array}{ll}
C_{i} & D_{\{i\} . .1}
\end{array}\right]\right)-I^{N} K_{N}\left[\begin{array}{ll}
C_{N} & D_{\{N\} .1}
\end{array}\right]\right)-r
\end{aligned}
$$

where $I^{i}=\left[0_{r_{i} \times\left(n+r^{i-1}\right)}^{\mathrm{T}} I_{r_{i}} 0_{r_{i} \times\left(r-r^{i}\right)}^{\mathrm{T}}\right]^{\mathrm{T}}$, with $r^{i}=\sum_{j=1}^{i} r_{j}, i \in \mathscr{N}$. Noting that for almost any $K \in \mathscr{K}$, the rank function in (6) gets its maximum, which can only be some constant integer less than $n+1$, we obtain, by using Lemma 2.3 w.r.t. $K_{N}$,

$$
\begin{aligned}
& \max \left\{\operatorname{rank}\left(\lambda I-A-B(\theta I-K D)^{-1} K C\right), K \in \mathscr{K}\right\} \\
& =\max \left\{\operatorname { m i n } \left\{\operatorname{rank}\left(\left[\begin{array}{ccc}
\lambda I-A & B_{\{1 \ldots \ldots N-1\}} & B_{N} \\
0 & \theta I_{r N-1} & 0
\end{array}\right]-\sum_{i=1}^{N-1} \tilde{I}^{i} K_{i}\left[\begin{array}{ll}
C_{i} & D_{\{i\} .}
\end{array}\right]\right),\right.\right. \\
& \left.\operatorname{rank}\left(\left[\begin{array}{cc}
\lambda I-A & B_{\{1, \ldots, N-1\}} \\
0 & \theta I_{r N-1} \\
C_{N} & D_{\{N\}\{1, \ldots, N-1\}}
\end{array}\right]-\sum_{i=1}^{N-1} I^{i} K_{i}\left[\begin{array}{ll}
C_{i} & D_{\{1\}\{1 \ldots, N-1\}}
\end{array}\right]\right), K \in \mathscr{K}\right\}-r^{N-1} \\
& =\min \left\{\operatorname { m a x } \left\{\operatorname{rank}\left(\left[\begin{array}{ccc}
\lambda I-A & B_{\{1 \ldots, N-1\}} & B_{N} \\
0 & \theta I_{r N-1} & 0
\end{array}\right]-\sum_{i=1}^{N-1} \tilde{I}^{i} K_{i}\left[\begin{array}{cc}
C_{i} & D_{\{i\}, A}
\end{array}\right]\right),\right.\right. \\
& \left.\operatorname{rank}\left(\left[\begin{array}{cc}
\lambda I-A & B_{\{1, \ldots, N-1\}} \\
0 & \theta I_{r N-1} \\
C_{N} & D_{\{N\}\{1, \ldots, N-1\}}
\end{array}\right]-\sum_{i=1}^{N-1} I^{i} K_{i}\left[\begin{array}{cc}
C_{i} & D_{\{i\}\{1 \ldots N-1\}}
\end{array}\right], K \in \mathscr{K}\right\}\right\}-r^{N-1},
\end{aligned}
$$

where $\tilde{I}^{i}=\left[0_{r_{i} \times\left(n+r^{i-1}\right)}^{\mathrm{T}} I_{r_{i}}\right]^{\mathrm{T}}$. Using Lemma 2.3 sequentially w.r.t. $K_{N-1}, \ldots, K_{1}$, we can finally get

$$
\max \left\{\operatorname{rank}\left(\lambda I-A-B(\theta I-K D)^{-1} K C\right), K \in \mathscr{K}\right\}=\min \left\{\operatorname{rank}\left[\begin{array}{cc}
\lambda I-A & B_{\mathscr{S}} \\
C_{\mathscr{S}^{\perp}} & D_{\mathscr{S}^{\perp} \mathscr{\mathscr { S }}}
\end{array}\right], \mathscr{S} \subset \mathscr{N}\right\} .
$$

This, together with (4) and (5), leads to the result.

Theorem 3.6 states that the determination of the geometric multiplicity $m_{\lambda}$ of some decentralized fixed mode $\lambda$ involves only the calculation of ranks of $2^{N}$ matrices. Actually, the proof of Theorem 3.6 shows that $m_{\lambda}$ can also be easily obtained by finding the rank of $\lambda I-A-B(I-K D)^{-1} K C$ for almost any $K \in \mathscr{K}$. 


\subsection{Determination of decentralized cycle index of GDS}

Theorem 3.7. For GDS (1), the decentralized output feedback cycle index is determined by

$$
\operatorname{cyc}(A, B, C, D, \mathscr{K})= \begin{cases}1 & \operatorname{DFM}(C, A, B, D, \mathscr{K})=\emptyset, \\ \max \left\{m_{\lambda}, \lambda \in \operatorname{DFM}(A, B, C, D, \mathscr{K})\right\} & \text { otherwise. }\end{cases}
$$

Proof. If $\operatorname{DFM}(A, B, C, D, \mathscr{K})=\emptyset, \quad$ then $\quad p_{\mathrm{f}}(s)=1, \quad p_{\mathrm{v}}(s, K)=\operatorname{det}\left(s I-A-B(I-K D)^{-1} K C\right)$. From Theorem 3.4, we know that for almost all $K \in \mathscr{K}, A+B(I-K D)^{-1} K C$ has $n$ distinct eigenvalues. Thus, $\operatorname{cyc}(A, B, C, D, \mathscr{K})=1$.

In the case of $\operatorname{DFM}(A, B, C, D, \mathscr{K}) \neq \emptyset$, for any $K \in \mathscr{K}$,

$$
\begin{aligned}
\operatorname{cyc}\left(A+B(I-K D)^{-1} K C\right) & =\max \left\{\operatorname{dim} \mathscr{X}_{K \lambda}, \lambda \in \operatorname{sp}\left(A+B(I-K D)^{-1} K C\right)\right\} \\
& \geq \max \left\{\operatorname{dim} \mathscr{X}_{K \lambda}, \lambda \in \operatorname{DFM}(A, B, C, D, \mathscr{K})\right\} \\
& \geq \max \left\{m_{\lambda}, \lambda \in \operatorname{DFM}(A, B, C, D, \mathscr{K})\right\} .
\end{aligned}
$$

On the other hand, since the intersection of any finite robust set is still robust, by Theorems $3.4-3.6$, we have, for almost all $K \in \mathscr{K}$,

$$
\operatorname{cyc}\left(A+B(I-K D)^{-1} K C\right)=\max \left\{m_{\lambda}, \lambda \in \operatorname{DFM}(A, B, C, D, \mathscr{K})\right\} .
$$

Thus, the result follows from Definition 3.2.

The main contribution of Theorem 3.7 is the characterization of the relation between the geometric multiplicities of decentralized fixed modes and the decentralized cycle index of a general proper system. To determine the decentralized cycle index, it is more convenient to compute $\operatorname{cyc}\left(A+B(I-K D)^{-1} K C\right)$ directly for an arbitrary $K \in \mathscr{K}$ without a prior knowledge on the decentralized fixed modes. This is suggested by the fact that, for almost all $K \in \mathscr{K}$,

$$
\operatorname{cyc}(A, B, C, D, \mathscr{K})=\operatorname{cyc}\left(A+B(I-K D)^{-1} K C\right) .
$$

\subsection{Examples}

The following example illustrates some of the above results.

Consider a general proper system

$$
\dot{x}=\left[\begin{array}{rrr}
2 & 0 & 0 \\
0 & 1 & 0 \\
0 & 0 & -1
\end{array}\right] x+\left[\begin{array}{l}
0 \\
0 \\
1
\end{array}\right] u_{1}+\left[\begin{array}{l}
1 \\
1 \\
0
\end{array}\right] u_{2}, \quad y_{1}=\left(\begin{array}{lll}
1 & 0 & 0
\end{array}\right) x, \quad y_{2}=\left(\begin{array}{lll}
0 & 1 & 0
\end{array}\right) x+d u_{1} .
$$

If $d=0$, it can be computed that

$$
p_{v}\left(s, k_{1}, k_{2}\right)=s-k_{2}-1, \quad \operatorname{DFM}=\{-1,2\}, \quad m_{-1}=m_{2}=1, \quad \operatorname{cyc}(A, B, C, D, \mathscr{K})=1 .
$$

Now, the system cannot be stabilized using decentralized controllers due to the existence of an unstable decentralized fixed mode of 2 .

In the case of $d \neq 0$,

$$
\begin{aligned}
& p_{\mathrm{v}}\left(s, k_{1}, k_{2}\right)=s^{2}-\left(d k_{1} k_{2}+k_{2}+3\right) s+d k_{1} k_{2}+2 k_{2}+2, \\
& \mathrm{DFM}=\{-1\}, \quad m_{-1}=1, \quad \operatorname{cyc}(A, B, C, D, \mathscr{K})=1 .
\end{aligned}
$$

The system now is decentralized stable. This shows the effect of the existence of the parameter $d$. 


\section{Conclusions}

In this paper, the algebraic properties of general control systems subject to decentralized output feedback are investigated. We introduce some new concepts of the decentralized output feedback variable polynomial, the decentralized output feedback cycle index of general proper systems, and the geometric multiplicities of decentralized fixed modes. It is shown that almost all decentralized output feedback laws can be used to make the zeros of DVP distinct, and disjoint from any given finite set of points on the complex plane. The results presented here, besides being important for understanding general proper decentralized control systems, are also very useful in the analysis and design of dynamical hierarchical control systems [11].

\section{References}

[1] M. Bocher, Introduction to Higher Algebra (Macmillan, New York, 1907).

[2] J.P. Corfmat and A.S. Morse, Decentralized control of linear multivariable systems, Automatica 12 (1976) $479-495$.

[3] E.J. Davison and T.N. Chang, Decentralized stabilization and pole assignment for general proper systems, IEEE Trans. Automat. Control 35 (1990) 652-664.

[4] E.J. Davison, W. Gesing and S.H. Wang, An algorithm for obtaining the minimal realisation of a linear time-invariant system and determining if a system is stabilizable-detectable, IEEE Trans. Automat. Control 23 (1978) 1048-1054.

[5] E.J. Davison and S.H. Wang, Properties of linear time-invariant multivariable systems subject to arbitrary output and state feedback, IEEE Trans. Automat. Control 17 (1972) 24-32.

[6] M.E. Sezer and D.D. Siljak, Structurally fixed modes, Systems Control Lett. 1 (1981) 60-64.

[7] A.F. Vaz and E.J. Davison, A measure for the decentralized assignability of eigenvalues, Systems Control Lett. 10 (1988) 191-199.

[8] S.H. Wang and E.J. Davison, On stabilization of decentralized control systems, IEEE Trans. Automat. Control 18 (1973) $473-486$.

[9] W.M. Wonham, Linear Multivariable Control: A Geometric Approach (Springer, New York, 1977).

[10] X.K. Xie and H.Y. Jing, Fixed modes of decentralized control systems, Acta Automat. Sinica 12 (1986) $185-189$.

[11] R. Yu and W.B. Gao, Minimal order dynamical co-operation controller for large scale systems, in: Symp. of Int. Conf. on Systems, Man and Cybernetics (Internat. Academic Press, Beijing, 1988) 1341-1344.

[12] R. Yu and W.B. Gao, Algebraic properties of decentralized control systems, Internat. J. Control 50 (1989) 81-88. 